\title{
Novel Micro-fabricated Chip With Micro-channels for In-situ Observation of Liquid Samples and Processes in TEM
}

\author{
E. Jensen $^{1,2}$, A. Burrows ${ }^{2}$, K. Mølhave ${ }^{1}$ \\ 1. Technical University of Denmark, DTU Nanotech, Kgs. Lyngby, Denmark. \\ 2. Technical University of Denmark, DTU Cen, Kgs. Lyngby, Denmark
}

In-situ nanoscale observation of liquid samples or processes at liquid-solid interfaces is an important tool for furthering the understanding of nanoscale processes. The current state-of-the-art systems used for in-situ transmission electron microscopy (TEM) of liquid samples mostly use a two-chip system[1,2,3], although there are notable exceptions [4,5]. These chips have thin electrontransparent membranes which allow imaging of the liquid trapped between them. The viewing area in this type of chip is quite large and subject to a relatively large bulging effect when introduced to the vacuum in a TEM[6]. While it is still possible to obtain excellent images, the bulging makes the total mass thickness of liquid imaged difficult to estimate. In this work we present a new chip for in-situ TEM of liquids which uses an electron transparent microchannel to contain the liquid. The well-defined dimensions of the channel makes it easier to estimate the bulging and therefore the mass thickness of the liquid imaged. As a proof-of-concept the chip was filled with liquid and then imaged in a TEM.

The chip was fabricated with a sacrificial etch method. A thin (50 nm thick) layer of low-stress silicon nitride $\left(\mathrm{SiN}_{\mathrm{x}}\right)$ was grown on a wafer. This was followed by a $1 \mu \mathrm{m}$ thick layer of poly-Si which functions as the sacrificial layer. The sacrificial layer was patterned by reactive ion etching (RIE) to form what will later be the channels, and then a conformal coating layer of $\mathrm{SiN}_{\mathrm{x}}$ was grown to encapsulate the silicon structures. Another RIE process was used to selectively thin the $\mathrm{SiN}_{\mathrm{x}}$ in the imaging area to create windows for improved resolution, and then RIE was used to open inlets to the silicon filled channel. Finally a KOH etch removed the poly-Si inside the channel leaving the $\mathrm{SiN}_{\mathrm{x}}$ shell. An overview and a scanning electron microscopy (SEM) image of the chip can be seen in Figure 1. After fabrication the bottom of the channel is $25 \mathrm{~nm}$ thick, the top is $25 \mathrm{~nm}$ thick in the thinned regions and $175 \mathrm{~nm}$ thick in the non-thinned regions. The channel is $4 \mu \mathrm{m}$ wide and $1 \mu \mathrm{m}$ tall. Some channels were left unetched and can then be used as polysilicon based heaters for increasing temperature in the imaging region.

The TEM chip was then filled with liquid containing $30 \mathrm{~nm}$ Au nanoparticles (see Figure 2) and sealed with an acrylic polymer varnish. The drying of the acrylic polymer consumed some of the liquid in the channel resulting in bubbles in the channel as revealed in the TEM images (see Figure 3 ), but otherwise the sample was stable for extended imaging.

This experiment shows that the $\mathrm{SiN}_{\mathrm{x}}$ channels can withstand the vacuum in the TEM as well as allow imaging of an enclosed liquid. The height of the channel allowed $1 \mu \mathrm{m}$ freedom of movement for diffusion and therefore the nanoparticles did not stay in focus long enough for clear in-focus images in the TEM. Future fabrication will reduce the height of the channel to improve resolution, reduce diffusion, and also to introduce electrical connection to the liquid in the channel for in-situ electrochemical measurements[7].

\author{
References \\ [1] N. de Jonge and F. Ross, Nature Nanotechnology 6(2011) 695-704 \\ [2] H. Zheng et al, Science 324 (2009) 1309-1312 \\ [3] J. E. Evans et al, Nano Letters 11 (2011) 2809-2813 \\ [4] J. M. Yuk et al, Science 336 (2012) 61-64 \\ [5] A. Radisic et al, J. Phys. Chem. B 110 (2006) 7862-7668
}


[6] J. Yang and O. Paul, Sensors and Actuators A 97-98 (2002) 520-526

[7] We acknowledge funding from the DFF-Sapere Aude project LiquidEM and DTU-CEN
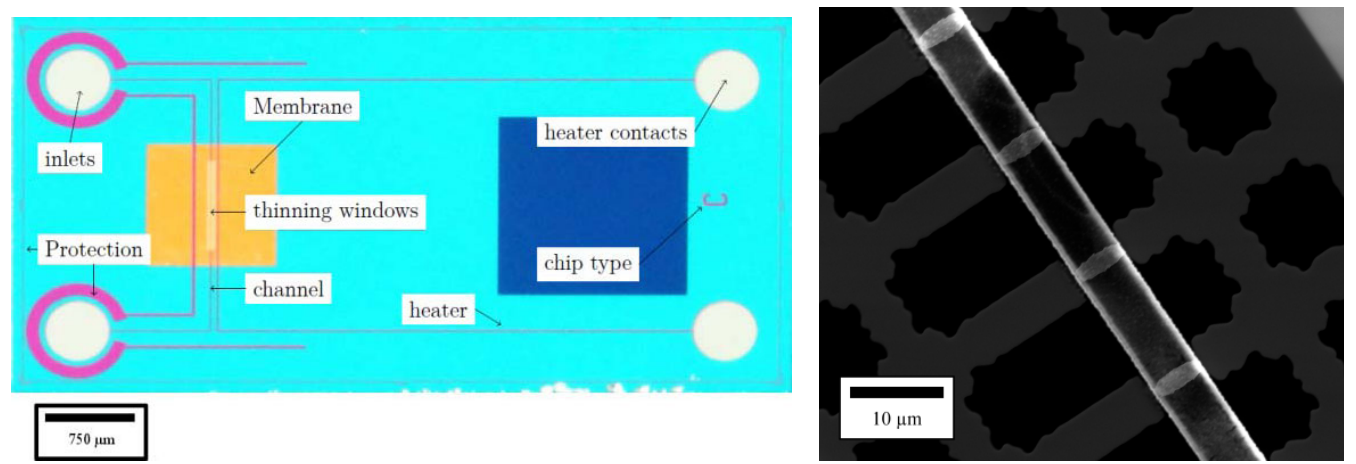

Figure 1. Optical microscope image of a TEM chip (left) and a SEM image of an empty channel with thinned areas (right). The optical image has labels indicating the two inlets to the channel, the two contact pads for the heater, the freestanding $\mathrm{SiN}_{\mathrm{x}}$ membrane that the micro-channel crosses and the areas where the $\mathrm{SiN}_{\mathrm{x}}$ has been thinned. The SEM image shows the microchannel running from the top left to bottom right. The darker areas along the channel are the areas where the $\operatorname{SiN}_{\mathrm{x}}$ has been thinned to $50 \mathrm{~nm}$. The $\mathrm{SiN}_{\mathrm{x}}$ is $200 \mathrm{~nm}$ thick everywhere else.
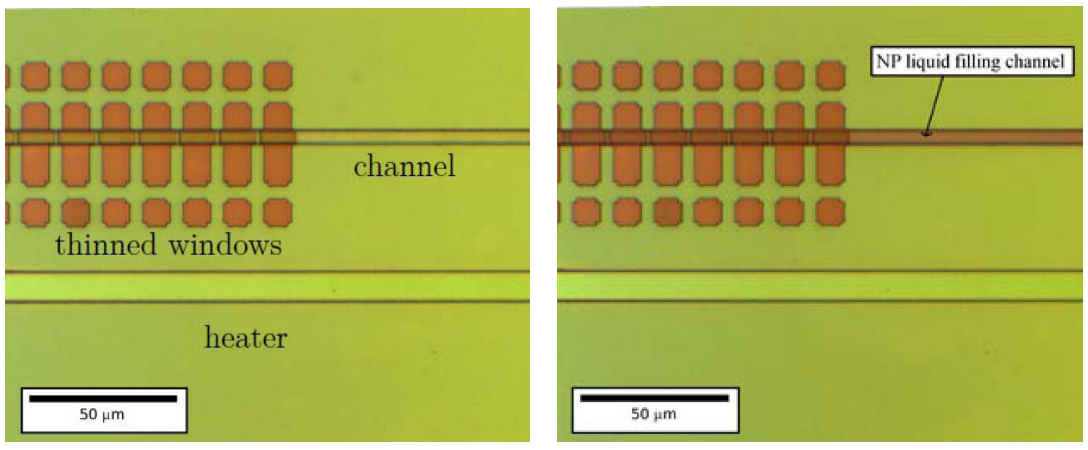

Figure 2. Optical microscope images of the TEM chip with micro-channel before (left) and after (right) the channel is filled. The substrate is the $\mathrm{SiN}_{\mathrm{x}}$ membrane the channel is fabricated on. The red areas are the areas where the $\mathrm{SiN}_{\mathrm{x}}$ has been thinned to $50 \mathrm{~nm}$. The channel runs perpendicular to these areas. Along the bottom of the image there is an empty channel $\mathrm{SiN}_{\mathrm{x}}$.

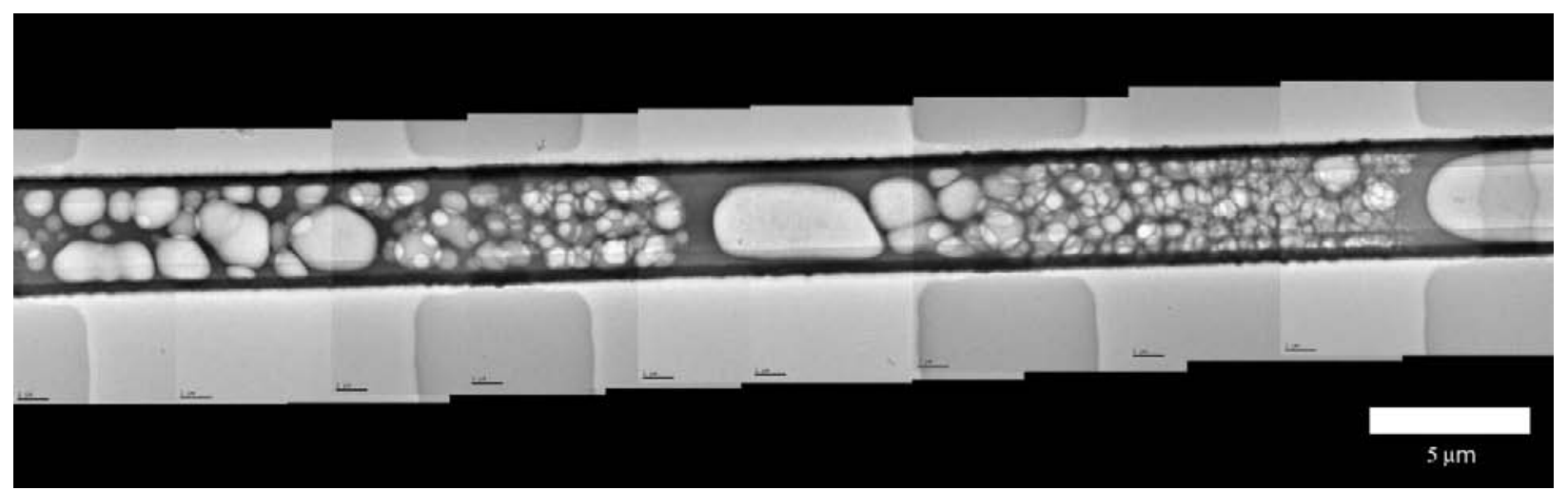

Figur 3. Composite image of multiple TEM images. The channel can be seen running through the center of the image. Inside the channel the dark-gray area is the liquid and the lighter areas are the bubbles. Outside the image the slightly darker areas indicate thick areas about $200 \mathrm{~nm}$ thick and the lighter areas are about $50 \mathrm{~nm}$ thick. 